\title{
m-Health no controle do câncer de colo do útero: pré-requisitos para o desenvolvimento de um aplicativo para smartphones
}

\author{
m-Health on the cervical cancer control: prerequisites for developing a \\ smartphone application

\section{m-Health en el control del cáncer de cuello uterino: requisitos previos para desarrollar una aplicación para teléfonos inteligentes}

Carolina Correia Bilotti | carolinac bilotti@hotmail.com

Centro Universitário Cesumar. Maringá (PR), Brasil.

Larissa Dalpiaz Nepomuceno | lari.dalpiaz@hotmail.com

Centro Universitário Cesumar. Maringá (PR), Brasil.

Gabriela Marengone Altizani | gabialtizani@gmail.com

Centro Universitário Cesumar. Maringá (PR), Brasil.

Regiane da Silva Macuch | rmacuch@gmail.com

Centro Universitário Cesumar. Maringá (PR), Brasil.

Tiago Franklin Rodrigues Lucena | tiagofranklin@gmail.com

Centro Universitário Cesumar. Maringá (PR), Brasil.

Flavio Bortolozzi | flavio.bortolozzi@unicesumar.edu.br

Centro Universitário Cesumar. Maringá (PR), Brasil.

Marcelo Picinin Bernuci | mbernuci@gmail.com

Centro Universitário Cesumar. Maringá (PR), Brasil.

\section{RESUMO}

Com o objetivo de fornecer informações sobre o câncer do colo do útero (CCU), usando canais de comunicação presentes nos smartphones, o estudo apresentado neste artigo caracterizou o padrão de uso desses canais e a preferência de recebimento de informações de 395 mulheres, foco do programa de rastreio do CCU do município de Maringá (PR). Entre essas mulheres, 89\% possuem celulares e $65 \%$ com acesso à internet. Entre as entrevistadas que utilizam a internet, $46 \%$ usam para acessar redes sociais, $45 \%$ para fazer pesquisas e 33\% para ler notícias. Entre as que utilizam as redes sociais, 55\% têm acesso ao Facebook e $56 \%$ ao WhatsApp. A porcentagem das que preferem receber informações por meio de panfletos é de $52 \%$ e, para 48\% delas, os médicos são os profissionais mais indicados para darem as informações. Embora haja significativa utilização dos canais de comunicação presentes nos smartphones, muitas mulheres ainda preferem receber informações sobre o CCU através dos métodos tradicionais de educação em saúde.

Palavras-chave: promoção da saúde; tecnologia biomédica; saúde pública; acesso à informação; atividades cotidianas. 


\section{ABSTRACT}

In order to provide information about cervical cancer using the communication channels present in the smartphones, the study presented in this article characterized the pattern of use of these channels and the preference of receiving information from 395 women who take part in in the cervical cancer screening program of the city of Maringá (PR).. 89\% of those women have cellular phones and $65 \%$ have access to the internet. Among those interviewed using the internet, $46 \%$ use to access social networking, $45 \%$ to research, and 33\% to read news. Among those who use social networkings, 55\% have access to Facebook and $56 \%$ to WhatsApp. Nevertheless, $52 \%$ prefer to receive information through pamphlets and for $48 \%$ of them doctors are the best professionals who give the information. Although there is significant use of the communication channels present in smartphones, many women still prefer to receive information about the cervical cancer through traditional methods of health education.

Keywords: health promotion; biomedical technology; public health; access to information; activities of daily life.

\section{RESUMEN}

Con el fin de proporcionar información sobre el cáncer de cuello uterino utilizando los canales de comunicación presentes en los teléfonos inteligentes, el estudio presentado en este artículo caracterizó el patrón de uso de estos canales y la preferencia de recibir información de 395 mujeres que participan en el programa de rastreio del cáncer de cuello uterino del municipio de Maringá (PR). El 89\% de esas mujeres tienen teléfonos celulares y el 65\% tienen acceso a Internet. Entre los entrevistados que utilizan internet, el $46 \%$ utiliza para acceder a redes sociales, el $45 \%$ a investigación y el $33 \%$ a noticias. Entre los que utilizan redes sociales, el $55 \%$ tiene acceso a Facebook y el 56\% a WhatsApp. Sin embargo, el 52\% prefiere recibir información a través de folletos y para el $48 \%$ de ellos los médicos son los mejores profesionales que dan la información. Aunque existe un uso significativo de los canales de comunicación presentes en los teléfonos inteligentes, muchas mujeres todavía prefieren recibir información sobre el cáncer cervical a través de los métodos tradicionales de educación sanitaria.

Palabras clave: promoción de la salud; tecnología biomédica; salud pública; acceso a la información; actividades cotidianas.

Contribuição dos autores:

Concepção e desenho do estudo - Carolina Correia Bilotti, Marcelo Picinin Bernuci, Regiane da Silva Macuch, Tiago Franklin Rodrigues Lucena, Flavio Bortolozzi.Aquisição, análise ou interpretação dos dados - Carolina Correia Bilotti, Larissa Dalpiaz Nepomuceno, Gabriela Marengone Altizani, Marcelo Picinin Bernuci, Regiane da Silva Macuch.Redação do manuscrito - Carolina Correia Bilotti, Larissa Dalpiaz Nepomuceno, Gabriela Marengone Altizani, Marcelo Picinin Bernuci, Regiane da Silva Macuch, Tiago Franklin Rodrigues Lucena

Revisão crítica do conteúdo intelectual - Marcelo Picinin Bernuci, Regiane da Silva Macuch, Tiago Franklin Rodrigues Lucena, Flavio Bortolozzi.

Declaração de conflito de interesses: Os autores declaram que não há quaisquer conflitos de interesse.

Fontes de financiamento: não há

Considerações éticas: Centro Universitário Cesumar CEP/Cesumar sob o parecer nº 1.359.854 em 10/12/2015

Histórico do artigo: Submetido: 25.nov.2016 | Aceito: 08.maio.2017 | Publicado: 30.jun.2017

Licença CC BY-NC atribuição não comercial. Com essa licença é permitido acessar, baixar (download), copiar, imprimir, compartilhar, reutilizar e distribuir os artigos, desde que para uso não comercial e com a citação da fonte, conferindo os devidos créditos de autoria e menção à Reciis. Nesses casos, nenhuma permissão é necessária por parte dos autores ou dos editores. 


\section{Introdução}

Com a reorganização do SUS, o modelo de assistência à saúde pública no Brasil sofreu profundas modificações. Houve um redirecionamento do padrão de atenção primária, valorizando a promoção da saúde, bem como ações preventivas e curativas de doenças ${ }^{1-2}$. Nesse sentido, a promoção da saúde se destaca por proporcionar ao indivíduo certo controle sobre sua saúde e sobre as ações que determinam a preservação da mesma, possibilitando que ele se empodere da saúde, aumentando sua capacidade de controle e gestão dos determinantes necessários para que doenças e agravos sejam evitados ${ }^{3-5}$. A necessidade do individuo se tornar protagonista dos cuidados em saúde acentua-se quando se percebe que, com o avanço do processo de envelhecimento populacional, as doenças crônicas crescem cada vez mais em detrimento das doenças infecciosas, que assolaram a população nos séculos passados. Diante desse fato, o câncer desponta como um importante problema de saúde pública mundial, justamente por faltar à maioria da população autonomia e sabedoria para lidar com o autocuidado, tendo, cada vez mais, doentes desinformados e despreparados para lidar com sua doença, o que dificulta a terapêutica e a condução do processo doença-terapia ${ }^{6}$.

Segundo dados da Organização Mundial da Saúde7, o câncer é um problema de saúde pública, especialmente entre os países em desenvolvimento. Nas próximas décadas, o impacto do câncer na população pode chegar a $80 \%$ dos mais de 20 milhões de casos novos estimados para $2025^{8}$. No Brasil, com base nos programas de registros pré-estabelecidos pelo governo, como os Registros Hospitalares de Câncer (RHC) e o Sistema de Informação sobre Mortalidade (SIM), fornecidos pelo Datasus, o câncer configura-se como uma condição em que há necessidade de planejamento e avaliação das ações de controle e prevenção. Dos tipos de câncer mais frequentes no mundo, o de pulmão lidera essa lista, com uma incidência de cerca de 1,8 milhão, seguido do câncer de mama (1,7 milhão), intestino (1,4 milhão) e próstata (1,1 milhão) . No sexo feminino, além do câncer de mama, o câncer do colo do útero (CCU) tem grande importância mundialmente, com incidência de $7,9 \%^{8}$. Além disso, é possível perceber que a incidência do câncer do colo do útero está muito relacionada com condições socioeconômicas menos favoráveis ${ }^{9}$, o que evidencia, de forma ainda mais clara, a importância de medidas mais eficazes e práticas no que tange à prevenção desse tipo de câncer.

Dessa forma, são cada vez mais importantes ferramentas que tornem o processo de prevenção do câncer mais acessível e tragam mais autonomia para o paciente. Com o ingresso progressivo e permanente da tecnologia na vida da população brasileira, dois modelos vêm despontando como estratégias a serem contempladas no processo de promoção e prevenção da saúde, o e-health e o m-health ${ }^{10}$. Tais denominações designam instrumentos de computação móvel, sensores médicos e dispositivos de comunicação de forma a expandir a cobertura e melhorar a eficácia do atendimento de saúde ${ }^{11}$. Para dados da União Internacional de Telecomunicações (ITU), já existem no mundo mais de cinco bilhões de usuários de rede sem fio, sendo que mais de 70\% deles vivem em países de baixa renda ou renda média e a ubiquidade dos dispositivos móveis, incluindo zonas mais remotas, desperta o interesse de uso dessas tecnologias no processo de disseminação de informação e promoção da saúde.

O smartphone é hoje um veículo de fácil acesso à internet e de relativo baixo custo que emprega aplicativos dos mais variados temas e desponta como uma nova alternativa a fim de melhorar o acesso da população à saúde, bem como à promoção do conhecimento acerca dos cuidados preventivos dos quais ela (população) deverá se encarregar. Nessa conjuntura, o câncer é responsável por um problema de saúde pública crescente no Brasil, ao mesmo tempo em que é uma das patologias nas quais a promoção da saúde e as medidas preventivas podem ser eficazes. Portanto, estudar maneiras de intensificar essas medidas tem sido de suma importância, e as tecnologias móveis podem auxiliar em tais melhorias, justamente pela presença constante de usuários da tecnologia e o aumento do tempo dedicado por elesà interação com tais 
dispositivos. No entanto, cabe reconhecer o universo da usuária e como ela gostaria de receber informações sobre o CCU.

Por consequência, torna-se de grande valia desenvolver estratégias para o empoderamento da saúde das mulheres baseadas no m-Health, em especial, em relação a doenças de potencial preventivo como o CCU, a fim de garantir o fortalecimento dos programas já existentes de controle desta enfermidade. No entanto, criar sistemas que estejam fora do universo e interesse dos usuários é um erro constante quando se pensa em inovação. Compreendendo que um aplicativo no smartphone, por exemplo, concorre com a atenção distribuída, e que muitos softwares apresentam problemas de concepção de interface por não terem sido centrados no universo das usuárias, e também que a pesquisa sobre o comportamento dos usuários ${ }^{12-13}$ é recomendação do processo de criação de sistemas interativos, objetivou-se neste estudo, caracterizar o perfil da população-alvo de um aplicativo a ser delineado para o fortalecimento do programa de prevenção do CCU. A caracterização sociodemográfica do perfil de acesso e uso às ferramentas de tecnologia da informação e comunicação, bem como o acesso a informações sobre os métodos preventivos são prérequisitos importantes a serem incorporados no desenvolvimento do aplicativo e podem ser decisivos no acesso a ele pela população em questão.

\section{Método}

Trata-se de um estudo descritivo do tipo transversal realizado no município de Maringá - PR, no qual foram aplicados questionários em mulheres entre os 20 e $>60$ anos de idade cadastradas nas 32 Unidades Básicas de Saúde (UBS) do município. Este estudo foi aprovado pelo comitê de ética do Centro Universitário Cesumar CEP/Cesumar sob o parecer $\mathrm{n}^{0}$ 1.359.854 em 10/12/2015.

Utilizou-se uma amostragem aleatória estratificada, já que a população de mulheres na faixa etária do estudo $(\mathrm{n}=99.608)$ dividia-se conforme as UBS, nas quais elas estavam cadastradas. Considerado no cálculo amostral o nível de significância de $\alpha=5 \%$ e o erro máximo admitido entre a estimativa e o valor real do parâmetro de $e=0,05$, o tamanho da amostra total necessário para estimar as características de interesse das pacientes foi de 443 mulheres. A amostra final consistiu em 395 mulheres, uma vez que 48 questionários não foram aplicados devido à microárea estar descoberta por motivo de afastamento ou demissão do agente comunitário de saúde (ACS) responsável.

Como em cada uma das UBS existem equipes de ACS responsáveis por atender, de modo domiciliar, as usuárias de uma determinada região, o total de mulheres incluídas no estudo foi distribuído de modo aleatório entre todas as ACS de cada UBS. Os ACS, por sua vez, distribuíram aleatoriamente o instrumento de pesquisa à quantidade de mulheres de cada faixa etária que foi determinada pelo sorteio aleatório. Dessa forma, a aplicação dos questionários foi realizada pelos ACS, respeitando a faixa etária, a microárea e a quantidade de questionários que receberam. Os ACS, nos dias das visitas domiciliares às mulheres sob o seu acompanhamento, indagavam se as mesmas tinham interesse em participar da pesquisa. Aquelas que aceitavam eram convidadas a responder o questionário e assinar o termo de consentimento livre e esclarecido. As mulheres que apresentavam dificuldade em responder, eram auxiliadas pelos ACS. Em data combinada, as pesquisadoras retornaram às UBS para buscar os questionários respondidos que, ao serem entregues às entrevistadas, tiveram o prazo médio de 20 dias para devolução. Cabe ressaltar que os ACS que tiveram dificuldade de aplicar o questionário ou se recusaram a aplicar sozinhos foram acompanhados pelas pesquisadoras em data estabelecida para nova visita às residências das mulheres para aplicação dos questionários.

Os dados coletados foram tabulados em planilha Excel. Os resultados foram analisados por meio de análise descritiva, com o auxílio do ambiente estatístico $\mathrm{R}$ ( $R$ Development Core Team) para a obtenção de tabelas de frequência. A porcentagem foi calculada dividindo-se a frequência absoluta pelo número de respondentes 
do questionário. Os comentários levaram em consideração as características e perfil de consumo e uso das tecnologias (com foco nas móveis), e essas características estão aplicadas nos itens de interface e desenho de informações endereçadas a essas mulheres em futuro aplicativo que funcionará como assistente pessoal.

\section{Resultados}

\section{Caracterização sociodemográfica}

Os dados referentes à caracterização sociodemográfica apresentam uma população com estado civil predominante casada $(60,35 \%)$, com ensino médio completo $(36,11 \%)$ e renda familiar preponderante de um a dois salários mínimos (58,84\%). A maior parte das entrevistadas $(84,09 \%)$ tem filhos, sendo que 31,31\% ficaram grávidas duas vezes. Quanto ao uso de anticoncepcionais, 64,39\% das mulheres já tomaram ou tomam e $84,36 \%$ não fazem uso de terapia hormonal. A maior porcentagem das entrevistadas (79,04\%) não fuma ou fumou. Entre essas mulheres, $62,37 \%$ possuem casa própria e moram com seu cônjuge (54,55\%) e filhos (53,28\%) (dados não mostrados).

\section{Caracterização da qualificação profissional e relação de trabalho}

Os dados referentes à caracterização da qualificação profissional e relação de trabalho apresentam uma quase maioria de mulheres que não exerce atividade remunerada (49,37\%). Portanto, as mesmas foram redirecionadas para o bloco $\mathrm{C}$ do questionário, não respondendo às perguntas sobre cargo/função atual $(48,61 \%)$, há quanto tempo trabalha na função atual $(52,66 \%)$, os turnos em que trabalha $(51,90 \%)$ e se fez capacitação para assumir o cargo (66,33\%). No que diz respeito às mulheres que exercem atividade remunerada, percebeu-se uma heterogeneidade em relação ao cargo ou à profissão exercida (30,89\%). $\mathrm{O}$ tempo de trabalho na profissão atual se concentrou entre um e 10 anos $(34,68 \%)$. Quanto ao turno de trabalho, houve um predomínio de mulheres que trabalham no período matutino e vespertino. Quando perguntado se as mesmas realizaram algum curso ou capacitação para atuarem no cargo que ocupam, 18,23\% disseram que não (dados não mostrados).

\section{Caracterização do tipo de uso das ferramentas de tecnologia da informação e comunicação (TIC)}

Os dados referentes à caracterização do tipo de uso das ferramentas de tecnologia da informação e comunicação são apresentados na Tabela 1. Embora 52,41\% das entrevistadas tenham afirmado que possuem computador pessoal, $36,96 \%$ não o utilizam e 36,71\% o utilizam há mais de cinco anos, sendo que $64,30 \%$ não precisa de ajuda para usá-lo. A maioria das mulheres $(89,11 \%)$ possuem celular há mais de cinco anos (63,54\%), sendo que 65,06\% têm celular com acesso à internet. O celular é predominantemente utilizado para fazer ligações (70,38\%) e receber ligações (65,57\%). O acesso à internet se dá principalmente através de Wifi pago $(56,46 \%)$ e o dispositivo mais utilizado para isso é o celular (50,63\%). A maioria das mulheres que acessam a internet não tem tempo fixo diário (40,25\%). Entre as entrevistadas que utiliza a internet, $54,18 \%$ possui e-mail, mas a maioria não o acessa (44,56\%) e usa o celular para acessar redes sociais (46,33\%), fazer pesquisas (44,81\%) e ler notícias (32,66\%). Entre as que utilizam as redes sociais, 54,68\% têm Facebook e 55,95\% Whatsapp. A porcentagem das entrevistadas que não usa nenhum recurso para comunicação por vídeo pela internet é de 75,44\% e entre os outros recursos tecnológicos, 34,68\% têm máquina fotográfica digital e 36,20\% possuem pendrive. 
Tabela 1. Frequências absolutas e relativas das características referentes ao tipo de uso das TIC

\begin{tabular}{|c|c|c|}
\hline Fatores & Frequência & $\%$ \\
\hline \multicolumn{3}{|c|}{ Você possui computador pessoal? } \\
\hline Não & 177 & $44,81 \%$ \\
\hline Não sei o que é & 5 & $1,27 \%$ \\
\hline Sim & 207 & $52,41 \%$ \\
\hline Não respondeu & 6 & $1,52 \%$ \\
\hline \multicolumn{3}{|c|}{ Há quanto tempo você utiliza computadores? } \\
\hline Não utilizo & 146 & $36,96 \%$ \\
\hline Menos de 1 ano & 5 & $1,27 \%$ \\
\hline De 1 a 3 anos & 33 & $8,35 \%$ \\
\hline De 3 a 5 anos & 35 & $8,86 \%$ \\
\hline Mais de 5 anos & 145 & $36,71 \%$ \\
\hline Não respondeu & 31 & $7,85 \%$ \\
\hline \multicolumn{3}{|c|}{ Você precisa de ajuda para usar o computador? } \\
\hline Não & 254 & $64,30 \%$ \\
\hline Sim & 87 & $22,03 \%$ \\
\hline Não respondeu & 54 & $13,67 \%$ \\
\hline \multicolumn{3}{|c|}{ Você possui celular? } \\
\hline Não & 32 & $8,10 \%$ \\
\hline Sim & 352 & $89,11 \%$ \\
\hline Não respondeu & 11 & $2,78 \%$ \\
\hline \multicolumn{3}{|c|}{ Há quanto tempo você utiliza celular? } \\
\hline Não utilizo & 11 & $2,78 \%$ \\
\hline Menos de 1 ano & 11 & $2,78 \%$ \\
\hline De 1 a 3 anos & 38 & $9,62 \%$ \\
\hline De 3 a 5 anos & 50 & $12,66 \%$ \\
\hline Mais de 5 anos & 251 & $63,54 \%$ \\
\hline Não respondeu & 34 & $8,61 \%$ \\
\hline \multicolumn{3}{|c|}{ Qual sua marca de celular? } \\
\hline Nenhuma & 10 & $2,53 \%$ \\
\hline Apple & 18 & $4,56 \%$ \\
\hline Samsung & 158 & $40,00 \%$ \\
\hline LG & 65 & $16,46 \%$ \\
\hline Motorola & 55 & $13,92 \%$ \\
\hline Nokia & 28 & $7,09 \%$ \\
\hline Sony & 6 & $1,52 \%$ \\
\hline Outra & 20 & $5,06 \%$ \\
\hline Não respondeu & 35 & $8,86 \%$ \\
\hline \multicolumn{3}{|c|}{ Você possui celular com acesso à internet? } \\
\hline Não & 106 & $26,84 \%$ \\
\hline Sim & 257 & $65,06 \%$ \\
\hline Não respondeu & 32 & $8,10 \%$ \\
\hline \multicolumn{3}{|c|}{ Para qual atividade você mais utiliza o celular* } \\
\hline Fazer ligação & 278 & $70,38 \%$ \\
\hline Receber ligações & 259 & $65,57 \%$ \\
\hline Enviar SMS & 131 & $33,16 \%$ \\
\hline Acessar a Internet & 175 & $44,30 \%$ \\
\hline
\end{tabular}


Tabela 1. Frequências absolutas e relativas das características referentes ao tipo de uso das TIC

(continuação)

\begin{tabular}{|c|c|c|}
\hline Fatores & Frequência & $\%$ \\
\hline \multicolumn{3}{|c|}{ Para qual atividade você mais utiliza o celular* (continuação) } \\
\hline Jogar & 48 & $12,15 \%$ \\
\hline Outras & 36 & $9,11 \%$ \\
\hline Não respondeu & 42 & $10,63 \%$ \\
\hline \multicolumn{3}{|c|}{ Como você acessa a internet na maioria das vezes* } \\
\hline Não acesso & 103 & $26,08 \%$ \\
\hline Wifi pago & 223 & $56,46 \%$ \\
\hline Rede discada & 12 & $3,04 \%$ \\
\hline Rede pública & 5 & $1,27 \%$ \\
\hline Wifi público & 19 & $4,81 \%$ \\
\hline Casa de amigos & 20 & $5,06 \%$ \\
\hline Outros & 18 & $4,56 \%$ \\
\hline Não respondeu & 20 & $5,06 \%$ \\
\hline \multicolumn{3}{|c|}{ Qual dispositivo você mais utiliza para acessar a internet* } \\
\hline Não acesso & 100 & $25,32 \%$ \\
\hline Notebook/PC & 97 & $24,56 \%$ \\
\hline Celular & 200 & $50,63 \%$ \\
\hline Computador de amigos & 4 & $1,01 \%$ \\
\hline Computador no trabalho & 13 & $3,29 \%$ \\
\hline Tablet & 10 & $2,53 \%$ \\
\hline Outros & 4 & $1,01 \%$ \\
\hline Não respondeu & 22 & $5,57 \%$ \\
\hline \multicolumn{3}{|c|}{ Quantas horas por dia você passa na Internet } \\
\hline Nenhuma & 120 & $30,38 \%$ \\
\hline Sem tempo fixo & 159 & $40,25 \%$ \\
\hline De 1 a 3 horas & 65 & $16,46 \%$ \\
\hline De 4 a 6 horas & 16 & $4,05 \%$ \\
\hline De 7 a 10 horas & 8 & $2,03 \%$ \\
\hline Mais de 10 horas & 7 & $1,77 \%$ \\
\hline Não respondeu & 20 & $5,06 \%$ \\
\hline \multicolumn{3}{|l|}{ Você possui e-mail? } \\
\hline Não & 158 & $40,00 \%$ \\
\hline Não sei o que é & 5 & $1,27 \%$ \\
\hline Sim & 214 & $54,18 \%$ \\
\hline Não respondeu & 18 & $4,56 \%$ \\
\hline \multicolumn{3}{|c|}{ Você acessa seu e-mail com qual frequência? } \\
\hline Não acesso & 176 & $44,56 \%$ \\
\hline Raramente & 104 & $26,33 \%$ \\
\hline Uma vez por dia & 14 & $3,54 \%$ \\
\hline Várias vezes por dia & 11 & $2,78 \%$ \\
\hline Todos os dias & 40 & $10,13 \%$ \\
\hline Uma vez na semana & 26 & $6,58 \%$ \\
\hline Uma vez por mês & 2 & $0,51 \%$ \\
\hline Não respondeu & 22 & $5,57 \%$ \\
\hline
\end{tabular}


Tabela 1. Frequências absolutas e relativas das características referentes ao tipo de uso das TIC

(continuação)

\begin{tabular}{|c|c|c|}
\hline Fatores & Frequência & $\%$ \\
\hline \multicolumn{3}{|l|}{ Você utiliza redes sociais?* } \\
\hline Não & 129 & $32,66 \%$ \\
\hline Facebook & 216 & $54,68 \%$ \\
\hline WhatsApp & 221 & $55,95 \%$ \\
\hline YouTube & 93 & $23,54 \%$ \\
\hline Twitter & 25 & $6,33 \%$ \\
\hline Google+ & 91 & $23,04 \%$ \\
\hline Linkedin & 9 & $2,28 \%$ \\
\hline My Space & 2 & $0,51 \%$ \\
\hline Outras & 5 & $1,27 \%$ \\
\hline Não respondeu & 13 & $3,29 \%$ \\
\hline \multicolumn{3}{|l|}{ Você utiliza a internet para* } \\
\hline Não utilizo & 114 & $28,86 \%$ \\
\hline Jogar & 50 & $12,66 \%$ \\
\hline Entrar em redes sociais & 183 & $46,33 \%$ \\
\hline Fazer pesquisas & 177 & $44,81 \%$ \\
\hline Ler notícias & 129 & $32,66 \%$ \\
\hline Assistir filmes & 53 & $13,42 \%$ \\
\hline Consultar portais do governo & 37 & $9,37 \%$ \\
\hline Se distrair & 96 & $24,30 \%$ \\
\hline Estudar & 73 & $18,48 \%$ \\
\hline Comprar & 45 & $11,39 \%$ \\
\hline Ler e-mails & 59 & $14,94 \%$ \\
\hline Outros & 38 & $9,62 \%$ \\
\hline Não respondeu & 14 & $3,54 \%$ \\
\hline \multicolumn{3}{|c|}{ Você utiliza alguns dos recursos a baixo para se comunicar por vídeo pela internet?* } \\
\hline Nenhum & 298 & $75,44 \%$ \\
\hline Skype & 41 & $10,38 \%$ \\
\hline Messenger Vídeo & 43 & $10,89 \%$ \\
\hline Hangout & 5 & $1,27 \%$ \\
\hline Outros & 11 & $2,78 \%$ \\
\hline Não respondeu & 19 & $4,81 \%$ \\
\hline \multicolumn{3}{|c|}{ Que outros equipamentos tecnológicos você possui?* } \\
\hline Nenhum & 137 & $34,68 \%$ \\
\hline Webcam & 31 & $7,85 \%$ \\
\hline Máquina fotográfica digital & 137 & $34,68 \%$ \\
\hline Filmadora & 23 & $5,82 \%$ \\
\hline Tablet & 68 & $17,22 \%$ \\
\hline Pendrive & 143 & $36,20 \%$ \\
\hline Mp3 & 36 & $9,11 \%$ \\
\hline Relógio digital & 39 & $9,87 \%$ \\
\hline Medidor de pressão/glicemia digital & 51 & $12,91 \%$ \\
\hline Outros & 13 & $3,29 \%$ \\
\hline Não respondeu & 14 & $3,54 \%$ \\
\hline
\end{tabular}

${ }^{*}$ A questão admitia mais de uma resposta. 


\section{Caracterização do conhecimento sobre o câncer do colo do útero (CCU) e a prevenção}

A Tabela 2 reúne os dados referentes à caracterização do conhecimento sobre o CCU e prevenção. Notase que a maior parte das mulheres entrevistadas informou saber o que é câncer $(94,94 \%)$ enquanto 86,58\% indicam que sabem o que é CCU e 53,67\% conhecem alguém que já teve CCU. O conhecimento sobre a existência dos exames preventivos é de $92,66 \%$. Grande parte possui vida sexual ativa $(73,67 \%)$ com um único parceiro $(73,92 \%)$. A parte predominante das mulheres $(81,27 \%)$ afirmou realizar, no mínimo, uma consulta com o ginecologista a cada dois anos e $88,35 \%$ das entrevistadas já realizou o exame de prevenção do CCU. Caso as mulheres tenham algum sangramento/corrimento, $76,71 \%$ delas procuram o posto de saúde. Em relação ao conhecimento sobre o que é HPV, 63,29\% afirmaram saber o que é e $80,28 \%$ referem saber da existência da vacina contra o HPV. A maioria das mulheres (83,54\%) buscam os resultados dos exames preventivos e $98,23 \%$ afirma saber que o SUS oferece exames preventivos e preservativos gratuitos.

Tabela 2. Frequências absolutas e relativas das características referentes ao conhecimento sobre o câncer do colo do útero

\begin{tabular}{|c|c|c|}
\hline Fatores & Frequência & $\%$ \\
\hline \multicolumn{3}{|l|}{ Você sabe o que é câncer? } \\
\hline Não & 17 & $4,30 \%$ \\
\hline Sim & 375 & $94,94 \%$ \\
\hline Não respondeu & 3 & $0,76 \%$ \\
\hline \multicolumn{3}{|c|}{ Você sabe o que é câncer do colo do útero? } \\
\hline Não & 47 & $11,90 \%$ \\
\hline $\operatorname{Sim}$ & 342 & $86,58 \%$ \\
\hline Não respondeu & 6 & $1,52 \%$ \\
\hline \multicolumn{3}{|c|}{ Você conhece alguém que teve câncer do colo do útero? } \\
\hline Não & 176 & $44,56 \%$ \\
\hline Sim & 212 & $53,67 \%$ \\
\hline Não respondeu & 7 & $1,77 \%$ \\
\hline \multicolumn{3}{|c|}{ Existem exames que previnem o surgimento do câncer do colo do útero? } \\
\hline Não & 23 & $5,82 \%$ \\
\hline Não sei o que é & 1 & $0,25 \%$ \\
\hline Sim & 366 & $92,66 \%$ \\
\hline Não respondeu & 5 & $1,27 \%$ \\
\hline \multicolumn{3}{|l|}{ Você tem vida sexual ativa? } \\
\hline Não & 98 & $24,81 \%$ \\
\hline Sim & 291 & $73,67 \%$ \\
\hline Não respondeu & 6 & $1,52 \%$ \\
\hline \multicolumn{3}{|l|}{ Quanto à sua vida sexual } \\
\hline Não possuo parceiro sexual & 89 & $22,53 \%$ \\
\hline Possuo um único parceiro sexual & 292 & $73,92 \%$ \\
\hline Possuo mais de um parceiro sexual & 3 & $0,76 \%$ \\
\hline Outros & 2 & $0,51 \%$ \\
\hline Não respondeu & 9 & $2,28 \%$ \\
\hline
\end{tabular}


Tabela 2. Frequências absolutas e relativas das características referentes ao conhecimento sobre o câncer do colo do útero

(continuação)

\begin{tabular}{|l|l|l|l|}
\hline Fatores & Frequência & $\%$ \\
\hline Você realiza ao menos uma vez a cada dois anos uma consulta com ginecologista? \\
\hline Não & 70 & $17,72 \%$ \\
\hline Sim & 321 & $81,27 \%$ \\
\hline Não respondeu & 4 & $1,01 \%$ \\
\hline Você realizou o exame de prevenção do câncer do colo do útero? & 40 & $10,13 \%$ \\
\hline Não & 449 & $88,35 \%$ \\
\hline Sim & 6 & $1,52 \%$ \\
\hline Não respondeu & 6 & \\
\hline
\end{tabular}

Caso você tenha corrimento e/ou sangramento espontâneo. Qual das alternativas a baixo corresponde à conduta que você teria?*

\begin{tabular}{|l|l|l|}
\hline Pesquiso na internet o que devo fazer & 23 & $5,82 \%$ \\
\hline Pergunto para uma amiga/familiar o que devo fazer & 12 & $3,04 \%$ \\
\hline Vou ao posto de saúde e marco uma consulta & 303 & $76,71 \%$ \\
\hline Procuro o pronto atendimento & 36 & $9,11 \%$ \\
\hline Vou à farmácia e pergunto qual remédio devo usar & 7 & $1,77 \%$ \\
\hline Outros & 21 & $5,32 \%$ \\
\hline Não respondeu & 18 & $4,56 \%$ \\
\hline
\end{tabular}

Você sabe o que é o papiloma vírus humano (HPV)?

\begin{tabular}{|l|l|l|}
\hline Não & 67 & $16,96 \%$ \\
\hline Não sei o que é & 75 & $18,99 \%$ \\
\hline Sim & 250 & $63,29 \%$ \\
\hline Não respondeu & 3 & $0,76 \%$ \\
\hline
\end{tabular}

Você sabe se existe uma vacina contra o HPV?

\begin{tabular}{|l|l|l|}
\hline Não & 73 & $18,48 \%$ \\
\hline Sim & 317 & $80,25 \%$ \\
\hline Não respondeu & 5 & $1,27 \%$ \\
\hline
\end{tabular}

Quando você realiza exames preventivos você busca os resultados com qual frequência?

\begin{tabular}{|l|l|l|}
\hline Sempre & 330 & $83,54 \%$ \\
\hline Quase sempre & 19 & $4,81 \%$ \\
\hline Às vezes & 16 & $4,05 \%$ \\
\hline Quase nunca & 3 & $0,76 \%$ \\
\hline Nunca & 18 & $4,56 \%$ \\
\hline Não respondeu & 9 & $2,28 \%$ \\
\hline
\end{tabular}

o SUS oferece gratuitamente exames preventivos para o câncer de colo de útero?

\begin{tabular}{|l|l|l|}
\hline Não & 3 & $0,76 \%$ \\
\hline Não sei o que é & 1 & $0,25 \%$ \\
\hline Sim & 388 & $98,23 \%$ \\
\hline Não respondeu & 3 & $0,76 \%$ \\
\hline O SUS oferece gratuitamente preservativo? & \multicolumn{2}{|l|}{} \\
\hline Não & 2 & $0,51 \%$ \\
\hline Sim & 388 & $98,23 \%$ \\
\hline Não respondeu & 5 & $1,27 \%$ \\
\hline
\end{tabular}

${ }^{*}$ A questão admitia mais de uma resposta. 


\section{Caracterização do acesso às informações sobre o câncer do colo do útero (CCU)}

Os dados referentes à caracterização do acesso às informações sobre o CCU são apresentados na Tabela 3. Grande parte das entrevistadas $(88,35 \%)$ refere já ter visto algum cartaz sobre o CCU e 64,56\% afirmam que o posto de saúde realiza palestras sobre como prevenir o CCU, mas a maioria (52,15\%) não participou destas. $71,65 \%$ das mulheresjá receberam panfletos sobre a prevenção doCCU.Aparte predominante das entrevistadas (91,90\%) sabeda campanha Outubro Rosa e 89,87\% referem já ter assistido a propagandas na TV sobre a importância de se realizar exames preventivos. A maioria das mulheres (70,38\%) já recebeu informações no posto de saúde sobre o risco de desenvolver CCU e 71,90\% referem já ter recebido orientações no posto sobre as formas de prevenção do CCU. Ao indagar sobre como as mulheres preferiam receber informações sobre a prevenção do CCU, 52,66\% afirmaram que preferem que seja por meio de panfletos e 48,35\% das entrevistas apontaram os médicos como o profissional mais adequado para esclarecer dúvidas sobre a doença.

Tabela 3. Frequências absolutas e relativas das características de acesso às informações sobre câncer do colo do útero

\begin{tabular}{|c|c|c|}
\hline Fatores & Frequência & $\%$ \\
\hline \multicolumn{3}{|c|}{ No posto de saúde do seu bairro, você já viu algum cartaz sobre câncer do colo do útero? } \\
\hline Não & 24 & $6,08 \%$ \\
\hline Não sei o que é & 13 & $3,29 \%$ \\
\hline Sim & 349 & $88,35 \%$ \\
\hline Não respondeu & 9 & $2,28 \%$ \\
\hline \multicolumn{3}{|c|}{ O posto de saúde do seu bairro já ofereceu alguma palestra sobre como prevenir o CCU? } \\
\hline Não & 45 & $11,39 \%$ \\
\hline Não sei o que é & 83 & $21,01 \%$ \\
\hline Sim & 255 & $64,56 \%$ \\
\hline Não respondeu & 12 & $3,04 \%$ \\
\hline \multicolumn{3}{|c|}{$\begin{array}{l}\text { Você já participou de alguma palestra sobre as formas de prevenção do CCU. Em qual desses } \\
\text { lugares?* }\end{array}$} \\
\hline Nunca participei & 206 & $52,15 \%$ \\
\hline No posto de saúde & 143 & $36,20 \%$ \\
\hline Na associação comunitária & 12 & $3,04 \%$ \\
\hline Na escola do(s) meu(s) filho(s) & 16 & $4,05 \%$ \\
\hline Na igreja/salão & 12 & $3,04 \%$ \\
\hline Na associação comercial & 4 & $1,01 \%$ \\
\hline Em uma faculdade/universidade & 20 & $5,06 \%$ \\
\hline Outros & 19 & $4,81 \%$ \\
\hline Não respondeu & 11 & $2,78 \%$ \\
\hline \multicolumn{3}{|c|}{ Você já recebeu algum panfleto sobre as formas de prevenção do CCU? } \\
\hline Não & 101 & $25,57 \%$ \\
\hline Sim & 283 & $71,65 \%$ \\
\hline Não respondeu & 11 & $2,78 \%$ \\
\hline \multicolumn{3}{|c|}{$\begin{array}{l}\text { Durante o mês de outubro em todo o Brasil ocorre a Campanha Outubro Rosa. Você sabe o que } \\
\text { significa essa campanha? }\end{array}$} \\
\hline Não & 23 & $5,82 \%$ \\
\hline Sim & 363 & $91,90 \%$ \\
\hline Não respondeu & 9 & $2,28 \%$ \\
\hline
\end{tabular}


Tabela 3. Frequências absolutas e relativas das características de acesso às informações sobre câncer do colo do útero

(continuação)

\begin{tabular}{|c|c|c|}
\hline Fatores & Frequência & $\%$ \\
\hline \multicolumn{3}{|c|}{ Você já viu algum comercial na TV sobre a importância de se realizar exames preventivos do CCU? } \\
\hline Não & 31 & $7,85 \%$ \\
\hline Sim & 355 & $89,87 \%$ \\
\hline Não respondeu & 9 & $2,28 \%$ \\
\hline \multicolumn{3}{|c|}{$\begin{array}{l}\text { Você já recebeu orientações no posto de saúde sobre os fatores de risco para o desenvolvimento do } \\
\text { CCU? }\end{array}$} \\
\hline Não & 71 & $17,97 \%$ \\
\hline Não frequenta & 35 & $8,86 \%$ \\
\hline Sim & 278 & $70,38 \%$ \\
\hline Não respondeu & 11 & $2,78 \%$ \\
\hline \multicolumn{3}{|c|}{ Você já recebeu orientações no posto de saúde sobre as formas de prevenção do CCU? } \\
\hline Não & 73 & $18,48 \%$ \\
\hline Não frequenta & 31 & $7,85 \%$ \\
\hline Sim & 284 & $71,90 \%$ \\
\hline Não respondeu & 7 & $1,77 \%$ \\
\hline \multicolumn{3}{|c|}{$\begin{array}{l}\text { Por quais das opções abaixo você gostaria de ser informada sobre as formas de prevenção do } \\
\text { câncer?* }\end{array}$} \\
\hline Panfleto & 208 & $52,66 \%$ \\
\hline Por mensagem de texto & 36 & $9,11 \%$ \\
\hline Por ligação telefônica & 30 & $7,59 \%$ \\
\hline Por e-mail & 45 & $11,39 \%$ \\
\hline Por WhatsApp & 82 & $20,76 \%$ \\
\hline Em grupos de redes sociais & 48 & $12,15 \%$ \\
\hline Por meio de palestras & 149 & $37,72 \%$ \\
\hline Outros & 18 & $4,56 \%$ \\
\hline Não respondeu & 10 & $2,53 \%$ \\
\hline \multicolumn{3}{|c|}{$\begin{array}{l}\text { Na sua opinião qual(s) do(s) profissional(s) abaixo possuem uma linguagem mais simples para } \\
\text { esclarecer dúvidas em relação às doenças?* }\end{array}$} \\
\hline Enfermeiros & 189 & $47,85 \%$ \\
\hline Médicos & 191 & $48,35 \%$ \\
\hline Professores & 45 & $11,39 \%$ \\
\hline Agentes de saúde & 162 & $41,01 \%$ \\
\hline Profissionais da saúde em geral & 129 & $32,66 \%$ \\
\hline Alguém que já teve a doença & 66 & $16,71 \%$ \\
\hline Outros & 17 & $4,30 \%$ \\
\hline Não respondeu & 9 & $2,28 \%$ \\
\hline
\end{tabular}

${ }^{*}$ A questão admitia mais de uma resposta.

\section{Discussão}

Este estudo se propôs, de forma geral, identificar o perfil de acesso às tecnologias móveis e a preferência da forma de obter informações a respeito da prevenção do câncer do colo do útero para que seja possível desenvolver estratégias que ampliem o empoderamento e autocuidado da saúde e, consequentemente, 
ocorram mais comportamentos preventivos, diminuindo a incidência ainda muito significativa do CCU. Os dados aqui obtidos confirmam que a maioria das mulheres sabe da existência do CCU, realiza exames preventivos, recebe panfletos e informações sobre a prevenção do CCU nas UBS e, no entanto, a prevalência e a mortalidade pelo CCU não têm diminuído. Portanto, o desenvolvimento de ferramentas de m-Health pode ser aplicado no fortalecimento das ações de controle do CCU baseado no empoderamento individual e coletivo em relação ao autocuidado.

Nesse sentido, é importante ressaltar que o empoderamento individual tem um caráter mais psicológico, voltado para o fortalecimento da autoestima e da capacidade de autocuidado enquanto o empoderamento comunitário está ligado à capacitação da comunidade para revindicar coletivamente seus direitos nas esferas deliberativas da sociedade ${ }^{14}$. Assim, para melhor uso do aplicativo pela população-alvo é necessária a incorporação de dinâmicas estabelecidas pelas redes sociais, combinadas aos métodos tradicionais de educação sobre saúde como panfletos, orientação de especialista e o desenvolvimento de ações coletivas em grupos face a face de mulheres para que a prevenção e a promoção da saúde em relação ao CCU sejam mais efetivas e eficazes como sugerido no presente estudo.

Em relação ao comportamento digital das entrevistadas, é possível perceber que o uso de computador como instrumento digital vem sendo substituído pelo uso de tecnologias móveis. Os avanços tecnológicos que ocorreram nos últimos tempos levaram ao surgimento de smartphones - dispositivos que incluem programas executados por um sistema operacional - que combinam funções de telefones celulares com tecnologia poderosa capaz de suportar aplicativos, acesso à Internet e conectividade sem fio com outros dispositivos. A saúde móvel (m-Health) encontra-se como uma alternativa possível atualmente graças à elevada inserção da internet na sociedade, ao aumento do uso dos smartphones e ao acesso de relativamente baixo custo à internet banda larga ${ }^{15}$. No entanto, o interesse em receber cartilhas e panfletos sobre o assunto ainda se configurou como um aspecto forte; desse modo, as ações de m-health devem estar alinhadas aos materiais educacionais já distribuídos pelo sistema de saúde, complementando ou reforçando os assuntos neles presentes. A cartilha impressa traz a informação de forma física e portá-las traz a sensação de posse da informação, não presente no uso de veículos de comunicação eletrônicos e digitais. De fato, a cartilha permite o arquivamento e o compartilhamento com os membros da família enquanto o smartphone condiciona um conteúdo mais individualizado e pontual. Estratégias de m-Health que reforçam programas de adesão a programas de autocontrole de saúde (diabetes, horários de medicamentos) ${ }^{16-17}$, controle de tabagismo ${ }^{18}$ ou envio de mensagens curtas com certa frequência para controle de vetores ${ }^{17-21}$ é um caminho que tem apontado bons resultados em outras pesquisas.

Dados da empresa Opus Software a respeito de estatística de uso celular no Brasil deixa claro essa tendência: no ano de 2015, o Brasil fechou com 191,8 milhões de acessos por banda larga móvel e, em contrapartida, os acessos por banda larga fixa não passaram de 25,4 milhões ${ }^{22}$. Isso se justifica pelo fato de que os telefones celulares são uma tecnologia mais barata e, consequentemente, mais acessível, o que torna sua abrangência cada vez maior como veículo de comunicação e informação. A importância do celular é tamanha que o Brasil está em sexto lugar no comércio de smartphones ${ }^{22}$. Além disso, cerca de 72 milhões de pessoas utilizam os celulares como meio de acesso à internet e, com isso, todos os benefícios que ela fornece como, por exemplo, a prática do e-commerce, ou seja, o comércio feito pela internet através de redes móveis e aplicativos de celular ${ }^{22}$. Nesse contexto, o m-health entra como meio de comunicação que torna o acesso à saúde mais simples e amplo, proporcionando maior autonomia do paciente a respeito do seu autocuidado, podendo assim monitorar sua saúde. Considera-se também que a internet é um dos principais meios pelos quais as pessoas buscam informações relacionadas à saúde $23-25$.

Como se sabe, o uso das tecnologias de informação e comunicação (TIC) é influenciado por diversos fatores, tais como os relacionados às condições sociais dos indivíduos e à comunidade em que vivem, o que pode determinar a diferença do uso da internet entre os gêneros ${ }^{26-27}$. Em dados obtidos sobre o uso 
da internet para acesso à informação sobre saúde, foi possível observar que dos $90 \%$ entrevistados que utilizam a internet para esse fim, o predomínio é do sexo feminino ( $89 \%$ da amostra) ${ }^{28}$. Essas informações evidenciam que as mulheres, comparadas aos homens, têm usado mais a internet voltada para a saúde. Outra característica importante é que o uso da internet entre homens e mulheres é de interesse, geralmente, diferente. Enquanto os homens procuram serviços mais diversificados e lúdicos, as mulheres buscam mais informações ${ }^{29}$, o que poderia justificar um envolvimento maior destas com informações relacionadas à saúde e ao autoconhecimento.

Como, atualmente, as mulheres possuem inúmeras atividades, tanto dentro quanto fora de casa, isso justifica os resultados da pesquisa, sobre a sua utilização da internet sem tempo fixo diário. Como também, o principal uso é para acessar redes sociais e fazer pesquisas, a importância de um meio como esse para que a mulher tenha uma proximidade maior com o autocuidado da saúde também fica evidente. Além disso, a crescente utilização dos dispositivos móveis para o cuidado do paciente tem conferido celeridade aos serviços assistenciais e aumentado o repositório de informações clínicas e, juntamente com a ampliação das redes de banda larga móvel e o fenômeno das redes sociais, surge um novo estilo de colaboração e suporte mútuo, no qual se verifica a necessidade de avaliar a utilização dessas tecnologias ${ }^{30}$.

O fato de grande parte das mulheres já se encontrar em estágios avançados do CCU, no momento do diagnóstico, piorando o prognóstico, pode ser explicado pela cobertura irregular dos exames de rastreio, no qual um segmento da população feminina se submete várias vezes ao longo dos anos enquanto outros nunca foram rastreados ${ }^{31}$. Ademais, o que se nota é uma estabilização nos índices de procura dos métodos de prevenção do câncer do colo do útero, como se pode observar comparando dados do presente trabalho com outras pesquisas feitas também no interior do Paraná, em que a prevenção do CCU é praticada por cerca $80 \%$ das mulheres ${ }^{32}$. O que justifica o fato paradoxal da mortalidade ainda significativa, mesmo com a cobertura preventiva alta, é que no Brasil as ações de prevenção não recebem uma caracterização de ações educativas, levando à falta de conscientização da população sobre a relevância do diagnóstico precoce, e à falta de definições dos serviços de saúde sobre o caminho a ser percorrido pela mulher, desde a queixa até o diagnóstico e tratamento especializado ${ }^{33}$. Aliada a essa conjuntura, está a falta de conhecimento científico da população brasileira a respeito dessa patologia, no qual as ideias estão focadas em conhecimentos populares, devido à falta de orientações teóricas repassadas pelos profissionais das unidades de saúde ${ }^{34}$, principalmente os agentes de saúde, que constituem importante intercâmbio de informações com essa população.

Desse modo, o CCU é ainda um grande problema para a saúde no Brasil posto que, ainda existem barreiras para um serviço de qualidade, como o desconhecimento das mulheres sobre o próprio corpo, falta de humanização no atendimento, e uma deficiente organização das redes de serviços para absorver a demanda de mulheres que necessitam de exames complementares e tratamento ${ }^{34}$. Além disso, por ser um câncer que produz poucos sintomas é evidenciado um comportamento comum de países subdesenvolvidos em que as condições socioeconômicas aliadas à desinformação, contribuem para que a população não procure assistência médica, caso não apresente sintomas ${ }^{35}$. Dessa forma, a mobilização dos meios de comunicação para uma melhor promoção da saúde relacionada com uma prevenção de qualidade do CCU tem se tornado cada vez mais importante. Como proposto pelo "Programa Viva Mulher" que serviu como base para a campanha 2002 de prevenção do câncer do colo do útero, é necessária a articulação de uma rede de comunicação com a mulher em que sejam preparadas ações como a produção de materiais de divulgação, que incluam folhetos para a mulher; cartazes de coleta, de fluxograma e de sensibilização da mulher. Todavia, observa-se na prática uma abordagem irregular mesmo com essas ações, o que pode ser explicado pelo fato de que os materiais de divulgação na abordagem da mulher para campanhas preventivas exploram conceitos que, apesar de terem o intuito de motivá-las, podem propor questões que não contribuem para que elas se envolvam na campanha ${ }^{36}$. 
Além disso, as campanhas de prevenção devem levar em consideração as especificidades culturais de cada região, os meios de comunicação utilizados para esse fim e a relação entre profissionais da saúde e comunidade. O que se observa, no entanto, é um subaproveitamento da rede pelas mulheres, o que pode justificar essa cobertura irregular, na qual quem precisa mais dos exames faz menos e vice-versa ${ }^{37}$. De acordo com o previsto pelo Manual técnico de prevenção ao $\mathrm{CCU}^{38}$, é preciso organizar o modelo de assistência na prevenção do câncer, promovendo facilidades na abordagem da mulher. Nesse contexto, surge a necessidade de novas ferramentas aliadas à tecnologia para proporcionar uma maior abrangência dos meios preventivos no âmbito da saúde pública, principalmente na área de oncologia em que o acompanhamento ${ }^{39}$, desses pacientes pode auxiliar na minimização dos efeitos nocivos à saúde dessa patologia, promovendo sua detecção precoce.

Diante dos dados expostos que determinam o aumento do uso das tecnologias móveis e os altos índices de CCU, acredita-se que o desenvolvimento de "apps" ou "aplicação móvel" - programa de computador projetado para ser executado em smartphones ou em outros dispositivos móveis com conteúdo capaz de promover mudanças no comportamento de risco e agregar mais conhecimento sobre o CCU, seja uma alternativa inovadora e eficaz para proporcionar a promoção da saúde em mulheres ${ }^{15}$. É sabido que o acesso às tecnologias e à inclusão digital não é total na população, porém está em constante crescimento. Portanto, infere-se que novas pesquisas relativas ao uso de celulares e o desenvolvimento de aplicativos como auxiliares na promoção da saúde devem ser efetivados. Estudos futuros randomizados e com tamanho amostral adequado ainda são necessários para melhor explorar a prevenção do câncer associado à capacidade dos smatphones e apps ${ }^{15}$. Em especial, aquelas que dizem respeito ao acompanhamento de exames preventivos do câncer e, sobretudo, do CCU. As tecnologias móveis são capazes de possibilitar o empoderamento do indivíduo acerca de sua saúde, ou seja, a autonomia para a tomada de decisões e, dessa maneira, diminuir a alta prevalência de câncer e outras doenças da população. Neste sentido, a conscientização das mulheres para o autocuidado, por meio das TIC móveis, pode ser de grande valia dentro dos programas de prevenção do CCU, uma vez que conscientização exige engajamento, para que então ocorra a transformação da realidade que tanto a Política de Promoção da Saúde preconiza ${ }^{40-41}$.

\section{Considerações finais}

Conforme demonstrado neste estudo, para umelhor uso do app pela população-alvo faz-se necessária a incorporação de dinâmicas pelas redes sociais, combinadas aos métodos tradicionais de educação sobre saúde (panfletos, orientação do especialista, e ações de promoção em grupo) além de subsidiar informações mais direcionadas ao hábito do autocuidado e da conscientização pelas mulheres, seja individual ou coletivamente.

\section{Referências}

1. Martins PC, Cotta RMM, Siqueira BR. Democracia e empoderamento no contexto da promoção da saúde: Democracia e empoderamento no contexto da promoção da saúde: possibilidades e desafios apresentados ao Programa de Saúde da Família. Physis [internet]. 2009 [citado em 2 jun. 2017];3(19): 679-694. Disponível em: http:// www.scielo.br/scielo.php?pid=S0103-73312009000300007\&script=sci abstract\&tlng=pt

2. Fertonani HP, Pires DEP, Biff D, Anjos SMD. Modelo assistencial em saúde: conceitos e desafios para a atenção básica brasileira. Ciência \& Saúde Coletiva [internet].2015 [citado em 2 jun. 2017];20(6):18691878. Disponível em: http://www.scielo.br/scielo.php?pid=S1413-81232015000601869\&script=sci abstract

3. Brasil. Ministério da Saúde. Secretaria de Políticas de Saúde. As cartas da promoção da saúde. Brasília: Ministério da Saúde; 2002. 
4. Taddeo PS, Gomes KWL, Caprara A, Gomes AMA, Oliveira GC, Moreira TMM. Acesso, prática educativa e empoderamento de pacientes com doenças crônicas. Ciência \& Saúde Coletiva [internet].2012 [citado em 2 jun. 2017]; 11(17): 2923-2930. Disponível em: http://www.scielo.br/scielo.php?script=sci arttext\& pid $=$ S1413-81232012001100009

5. Mascarenhas NB, Melo CMM, Fagundes NC. Produção do conhecimento sobre promoção da saúde e prática da enfermeira na Atenção Primária. Rev Bras Enferm [internet]. 2012 [citado em 2 jun. 2017]; 65(6):991-999. Disponível em: http://www.scielo.br/pdf/reben/v65n6/a16v65n6.pdf

6. Carvalho SR, Gastaldo D. Promoção à saúde e empoderamento: uma reflexão a partir das perspectivas crítico-social pós-estruturalista. CiêncSaúde Coletiva [internet].2007[citado em 2 jun. 2017]; 2(13):.2029-2040.Disponível em: http://www.scielo.br/scielo.php?script=sci_arttext\&pid $=$ S1413-81232008000900007

7. World Health Organization (WHO). M-Health: New horizons for health through mobile technologies. Geneva: WHO; 2011. Disponível em: http://www.who.int/goe/publications/goe mhealth web.pdf

8. Instituto Nacional de Câncer José Alencar Gomes da Silva (Inca). Estimativa 2016 de Incidência do Câncer no Brasil. Rio de Janeiro: Inca; 2015. Disponível em: http://www.inca.gov.br/estimativa/2016/ estimativa-2016-v11.pdf

9. Stewart BW,Wild CP, editores. World cancer report. Geneva: WHO; 2014. Disponível em: http:// publications.iarc.fr/Non-Series-Publications/World-Cancer-Reports/World-Cancer-Report-2014.

10. Salvi P. Telemedicina: saída para redução de custos e melhoria na qualidade do atendimento na saúde brasileira. [Internet]. Convergecom (SP); 2013[citado em 2 jun 2017]. Disponível em: http:// convergecom.com.br/portal/telemedicina-saida-para-reducao-de-custos-e-melhoria-na-qualidade-doatendimento-na-saude-brasileira

11. Instituto do Coração (Incor), Laboratório de Arquitetura e Redes de Computadores (Larc), Ericsson Telecomunicações SA. Mobile Health uma solução para melhorar a qualidade da saúde preventiva no Brasil. I Workshop Ibero-Americano de Sistemas Interoperáveis em Saúde; 2014.Ribeirão Preto: Incor; Larc; 2014. Disponível em: http://ciis.fmrp.usp.br/orah/files/iasis2014/Sessao6 mhealth_IASIS.pdf.

12. Dourish P, Button G. On technomethodology foundational relationships between ethnomethodology and system design. Human Comp Inter [internet].1998 [citado em 2 jun 2017];13(4):395-432. Disponível em: http://dl.acm.org/citation.cfm?id=1462996.

13. Viller S; Sommerville I. Social analysis in the requirements engineering process: from ethnography to method. IEEE Comput Soc [internet]. 1999 [citado em 2 jun 2017]; p.6-13. Disponível em: http:// ieeexplore.ieee.org/lpdocs/epic03/wrapper.htm?arnumber $=777980$

14. Kleba ME, Wendausen A. Empoderamento: processo de fortalecimento dos sujeitos nos espaços de participação social e democratização política. Saúde e sociedade [internet].2006 [citado em 2 jun 2017]; 18(4):733-743. Disponível em: http://www.scielo.br/scielo.php?pid=S0104$\underline{12902009000400016 \& \text { script=sci abstract\&tIng=pt. }}$

15. Coughlin S, Thind H, Liu B, Jacobs M, Champagne N, Massey RI. Mobile Phone Apps for Preventing Cancer Through Educational and Behavioral Interventions: State of the Art and Remaining Challenges. JMIR Mhealth Uhealth [Internet]. 2016 [citado em 2 jun 2017]; 4(2): e69. Disponível em: https://www. ncbi.nlm.nih.gov/pubmed/27242162.

16. Cafazzo, JA, Casselman M, Haming N, Katzman DK, Palmert MR. Design of an mHealth app for the selfmanagement of adolescent type 1 diabetes: a pilot study. J Med Internet Res[internet]. 2012 [citado em 2 jun 2017]; 14(3):e70.Disponível em: https://www.ncbi.nlm.nih.gov/pubmed/22564332

17. Stoner SA, Hendershot, CS. A randomized trial evaluating an mHealth system to monitor and enhance adherence to pharmacotherapy for alcohol use disorders. Addiction Science \& Clinical Practice [internet],2012 [citado em 2 jun 2017];7(1):9. Disponível em: http://www.pubmedcentral.nih.gov/ articlerender.fcgi? artid $=3507635 \&$ tool=pmcentrez\&rendertype $=$ abstract

18. Free $\mathrm{C}$, Knight R, Robertson $\mathrm{S}$ et al. Smoking cessation support delivered via mobile phone text messaging (txt2stop): a single-blind, randomised trial. Lancet [internet],2011 [citado em 2 jun 2017];378(9785):49-55. Disponível em: https://www.ncbi.nlm.nih.gov/pmc/articles/PMC3143315/

19. Cormick G, Kim NA, Rodgers A et al. Interest of pregnant women in the use of SMS (short message service) text messages for the improvement of perinatal and postnatal care. Reprod Health [internet], 2012 [citado em 2 jun 2017]; 9(1):9-15. Disponível em: http://www.reproductive-health-journal.com/ content/9/1/9 
20. KlasnjaP, Pratt W. Healthcare in the pocket: Mapping the space of mobile-phone health interventions. J Biomed Inform [internet], 2012 [citado em 2 jun 2017]; 45(1):184-198. Disponível em: http:// linkinghub.elsevier.com/retrieve/pii/S1532046411001444

21. Siedner MJ, Santorino D, Lankowski AJ et al. A combination SMS and transportation reimbursement intervention to improve HIV care following abnormal CD4 test results in rural Uganda: a prospective observational cohort study. BMC Medicine [internet], 2015; 13(1):160-173. Disponível em: http://www. biomedcentral.com/1741-7015/13/160

22. Opus Software. Estatísticas de uso de celular no Brasil [internet]. São Paulo: Opus, 2016 [citado em 2 jun 2017]. Disponível em: http://www.opus-software.com.br/estatisticas-uso-celular-brasil.

23. Morahan-Martin, JM. How internet users find, evaluate, and use online health information: a crosscultural review. CyberpsychologyBehav [internet] 2004 [citado em 2 jun 2017]; 7(5):497-510. Disponível em: https://www.ncbi.nlm.nih.gov/pubmed/15667044

24. Oravec JA. On the proper use of the Internet: self-help medical information and on-line health care. J Health Soc Policy [internet], 2001; 14(1):37-60. Disponível em: http://www.haworthpress.com/store/EText/View EText.asp?sid=GG4D3RXQAEVW9M2VFUWQ0F7SM9XG87M6\&a $=3 \& s=J 045 \& \mathrm{v}=14 \& \mathrm{i}=1 \& \mathrm{fn}=\mathrm{J}$ 045v14n01 03/nhttp://pdfserve.informaworld.com/711770 915549761 904525920.pdf

25. Usui N, Kamiyama M, Tani G, Kanagawa T, Fukuzawa M. Use of the medical information on the internet by pregnant patients with a prenatal diagnosis of neonatal disease requiring surgery. Pediatr Surg Int [internet], 2011 [citado em 2 jun 2017]; 27(12):1289-1293. Disponível em: https://www.ncbi.nlm.nih. gov/pubmed/21833721.

26. Ribeiro LCQ, Salata A, Costa L, Ribeiro MG. Desigualdades digitais: Acesso e uso da internet, posição socioeconómica e segmentação espacial nas metrópoles brasileiras. Analis Soc [internet], 2013 [citado em 2 jun 2017]; 207(48):288-320. Disponível em: http://analisesocial.ics.ul.pt/documentos/AS 207 d02.pdf

27. Simões MJ, Heras SL, Augusto A. Gênero e tecnologias da informação e da comunicação no espaço doméstico: não chega ter, é preciso saber, querer e poder usar. Configurações. Rev Soc [Internet], 2011 [citado em 2 jun 2017]; 8:155-172. Disponível em: https://configuracoes.revues.org/831

28. Moretti FA, Oliveira VE, Silva EMK. Access to health information on the internet: a public health issue. Rev Assoc Med Bras [Internet], 2012 [citado em 2 jun 2017]; 58(6):650-658. Disponível em: https:// www.researchgate.net/publication/233948794 Access to health information on the internet A public health issue

29. Silva AG, Olinto G. Diferenças de gênero no uso das tecnologias da informação e da comunicação: um estudo na biblioteca parque de Manguinhos. XVI Encontro Nacional de Pesquisa em Ciência da Informação; 2015; UFPB. João Pessoa: UFPB; 2015. Disponível em: http://www.ufpb.br/evento/ti/ocs/ index.php/enancib2015/enancib2015/paper/viewFile/3033/1068

30. Andrade MA, Prestes DP, Varani ML. TI Móvel na Saúde: privacidade, confidencialidade e segurança. Bras Eng Bioméd, 2014; 1(1):2314-2317.

31. Santos RS, Melo ECP, Santos KM. Spatial analysis of the indicators agreed for screening cervix cancer in Brazil. Texto ContEnf[Internet], 2012 [citado em 2 jun 2017]; 21(4):800-810. Disponível em: http:// www.scielo.br/scielo.php?pid=S0104-07072012000400010\&script=sci arttext\&tlng=en

32. Bim CR, Pelloso SM, Carvalho MDB. Diagnóstico precoce do câncer de mama e colo uterino em mulheres do município de Guarapuava, PR, Brasil. Rev Esc Enferm Usp [Internet],2010 [citado em 2 jun 2017]; 4(44): 940-946. Disponível em: http://www.scielo.br/pdf/reeusp/v44n4/12.pdf.

33. Pelloso SM, Carvalho MDB, Higarashi IH. Conhecimento das mulheres sobre o câncer cérvico-uterino. Acta Scientiarum Health Scienc[Internet], 2004 [citado em 2 jun 2017]; 26(2):319-324. Disponível em: https://www.nescon.medicina.ufmg.br/biblioteca/imagem/0261.pdf.

34. Santos MS, Macedo APN, Leite MAG. Percepção de usuárias de uma unidade de saúde da família acerca da prevenção do câncer do colo do útero. Rev. Aps. 2010; 13(3): 310-319.

35. Andrade MS, Almeida MMG, Araujo TM. Fatores associados à não adesão ao Papanicolau entre mulheres atendidas pela Estratégia Saúde da Família em Feira de Santana, Bahia, 2010. Epidemiol. Serv. Saúde. 2014; 1(23):111-120.

36. Cruz LMB, Loureiro RP. A comunicação na abordagem preventiva do câncer do colo do útero: importância das influências histórico-culturais e da sexualidade feminina na adesão às campanhas. Saúde Soc. [Internet]. 2008 [citado em 2 jun 2017]; 17(2): 120-131. Disponível em: http://www.scielo. br/scielo.php?script=sci arttext\&pid=S0104-12902008000200012 
37. Pontes FP. Adesão das mulheres ao exame de prevenção do câncer de colo uterino: uma visão integrativa [trabalho de conclusão de curso]. Conselheiro Lafaiete: Universidade Federal de Minas Gerais; 2012.

38. Brasil. Ministério da Saúde. Prevenção do câncer do colo do útero: Manual Técnico. Brasília: Ministério da Saúde; 2002. Disponível em: http://bvsms.saude.gov.br/bvs/publicacoes/inca/manua_assistencia.pdf.

39. Soares AB. Modelagem de um aplicativo m-Health para auxilio ao diagnóstico e cuidados em pacientes com fadiga oncológica.[dissertação]. Campina Grande: Universidade Estadual da Paraíba; 2013. Disponível em: http://dspace.bc.uepb.edu.br/jspui/bitstream/123456789/2294/1/PDF\%20-\%20 Adilson\%20Barros\%20Soares.pdf.

40. Freire P. Pedagogia do oprimido. Rio de Janeiro: Paz e Terra; 1983.

41. Brasil. Ministério da Saúde. Secretaria de Vigilância em Saúde. Secretaria de Atenção à Saúde. Política Nacional de Promoção da Saúde: PNPS: revisão da Portaria MS/GM no 687, de 30 de março de 2006. Brasília: Ministério da Saúde; 2015. 